\title{
Equitable Distribution in a Three Players Problem
}

\author{
Marek Szopa ${ }^{1}$ \\ 1 Institute of Physics, University of Silesia in Katowice, Poland, marek.szopa@us.edu.pl
}

\begin{abstract}
Jazz band is a 3 player superadditive game in characteristic function form. Three players have to divide the payoff they can get, while being in a grand coalition, provided their individual and duo coalitions payoffs are known. Assumptions of individual and collective rationality lead to the notion of the core of the game. We discuss offers that cannot readily be refused [OCRR] as the solutions of the game in case of an empty core, when duo coalitions are the best options but only for two out of three players. The experiment shows that even in case of an empty core the most probable results are three-way coalitions and the share of the weakest player usually exceeds his OCRR. The Shapley value is introduced and its fairness is discussed as it lies at the side of the core while, on the other hand, the nucleolus lies exactly at the center of the core. We conclude that, in spite of that, the Shapley value is the best candidate for a fair sharing solution of the jazz band game and other similar games as, opposite to the other values, it is dependent both on individual and duo coalitions payoffs.
\end{abstract}

Keywords: game theory; core of the game; Shapley value, nucleolus

\section{Introduction}

The cooperative game approach to fair division of goods is widely accepted to be the most promising way of deriving the problem. Different fairness concepts have been developed so far. The best known one is the proportionality law. If bank goes bankrupt, the customers who entrusted their savings to that bank are paid in proportion to amounts they had saved in the bank. This idea is well rooted in our culture because of Aristotle (Young, 1994). For proportionality to be workable the good must be divisible and the extend of each player's entitlement must be expressible in a common metric. But even when both conditions are met, proportionality may not be as compelling as it seems. Consider e.g. a situation where players do not have a single measure to draw their claims because their status quo depends on the temporary coalition they can form. 
It was von Neumann and Morgenstern (Von Neumann \& Morgenstern, 1944) who first considered the class of games in a characteristic function form, where players could form coalitions and opportunities available to each coalition could be described by a single number. This characteristic function model assumes that: the utilities are fully transferable among players, the possibilities accessible to a coalition of players can be assessed without reference to the players not included in the coalition and players are free to make arbitrary coalitions in any way agreed by the members (Roth, 1988). In recognition of the importance of the first assumption, these games are sometimes called transferable utility games.

The best known concepts of cooperative solutions are the core, the Shapley value and the nucleolus. In the present paper we discuss the notions in context of well known jazz band game introduced by Peyton Young. The example was described in (Malawski, Wieczorek, \& Sosnowska, 1997) without reference to the original source. (In my private communication with Young he could not remember that source either.)

\section{Jazz band game}

Three artists: a singer, a pianist and a drummer play together in a nightclub in Montmartre. They earn 1000 FF (French francs) per night, provided they perform together. But they often play in pairs or even individually in that club. It is known that their earnings in pairs are: the singer and the pianist $-800 \mathrm{FF}$, the pianist and the drummer $-650 \mathrm{FF}$ and the singer and the drummer $-500 \mathrm{FF}$. If they play individually the singer earns $200 \mathrm{FF}$ and the pianist $300 \mathrm{FF}$, whereas their colleague the drummer is not able to earn anything individually. The payroll is summarized in Table 1.

Table 1

The players' payroll in the jazz band game

\begin{tabular}{|l|c|c|c|c|c|c|c|}
\hline Players: & $\mathrm{S}$ & $\mathrm{P}$ & $\mathrm{D}$ & $(\mathrm{S}, \mathrm{P})$ & $(\mathrm{S}, \mathrm{D})$ & $(\mathrm{P}, \mathrm{D})$ & $(\mathrm{S}, \mathrm{P}, \mathrm{D})$ \\
\hline Earnings: & 200 & 300 & 0 & 800 & 500 & 650 & 1000 \\
\hline
\end{tabular}

A natural problem is the question how they should divide their earnings in case of any joint performances. A naive answer could be that their earnings when playing in duo should be proportional to single payoffs. This would give the following division: $320 \mathrm{FF}$ for the singer and $480 \mathrm{FF}$ for the pianist. However, this idea should be rejected since the drummer part in duo 
should then always be zero. Common sense leads us to the conclusion that players should not accept any division that yields a share which is smaller than the share they could get individually. This rule will be defined below as individual rationality.

In case of the trio the proportional division is even more obscure how to weigh individual and duo payoffs in proportion? Here the common sense rule, called the collective rationality, should ensure that the division in trio should ensure that each two players will gain at least the amount they are able to earn when playing in duo. Otherwise the players have no incentive to search for the grand coalition (trio). This leads us to the notion of a core of the problem. The core of the division problem is the set of payoffs that obey the rules of individual and collective rationality. In other words, a core division is such that gives every party, and every coalition of parties, an incentive to participate.

\section{The core of the game}

To define the core precisely, let us define the set of all players by $N=\left\{p_{1}, p_{2}, \ldots, p_{n}\right\}$, where $n \in \mathbb{N}$ is the total number of players. The coalition of players is $C \subseteq N$ i.e. an arbitrary subset of the set of all players. The characteristic function is the function $\nu: 2^{N} \rightarrow \mathbb{R}$, which for any subset $C \subseteq N$ returns a number $\nu(C)$, which is the payoff of the coalition $C$, i.e. the amount that the players in $C$ could earn if they formed a coalition. The payoff of the empty coalition is zero $\nu(\emptyset)=0$. The game is called superadditive provided $\nu(C \cup T) \geq \nu(C)+\nu(T)$, where $C, T \subseteq N$ are two disjoint i.e. $C \cap T=\emptyset$ coalitions.

In case of the jazz band game $n=3$ and the coalitions are: $\emptyset,\{S\}$, $\{P\},\{D\},\{S, P\},\{S, D\},\{P, D\},\{S, P, D\}$. The characteristic function is defined by:

$$
\begin{aligned}
& \nu(\emptyset)=0 \\
& \nu(S)=200 \quad \nu(P)=300 \quad \nu(D)=0 \\
& \nu(S, P)=800 \quad \nu(S, D)=500 \quad \nu(P, D)=650 \\
& \nu(S, P, D)=1000
\end{aligned}
$$

Note that the jazz band game is superadditive. The imputation is an $n$ tuple $\left(x_{1}, x_{2}, \ldots, x_{n}\right)$ which sums up to the payoff of the grand coalition $\sum_{i=1}^{n} x_{i}=\nu(N)$, which obeys the individual rationality condition $x_{i} \geq \nu\left(p_{i}\right)$. 
The core of the game is the set of all imputations, which obey collective rationality i.e.

$$
\sum_{p_{i} \in C} x_{i} \geq \nu(C)
$$

for any coalition $C \subseteq N$ containing at least two players. The core is therefore a set of all imputations that are acceptable for all possible coalitions. The idea of collective rationality means that the imputation can be in the core if the members of a given coalition $C$ are getting the total payoff that is at least as high as the payoff guaranteed by the characteristic function.

In case of the jazz band game the individual rationality means that $x_{S} \geq 200, x_{P} \geq 300$ and $x_{D} \geq 0$. The core is the set of all imputations that, apart from the individual rationality, obey the collective rationality, that in this case means that $x_{S}+x_{P} \geq 800, x_{S}+x_{D} \geq 500, x_{P}+x_{D} \geq 650$, see Fig. 1.

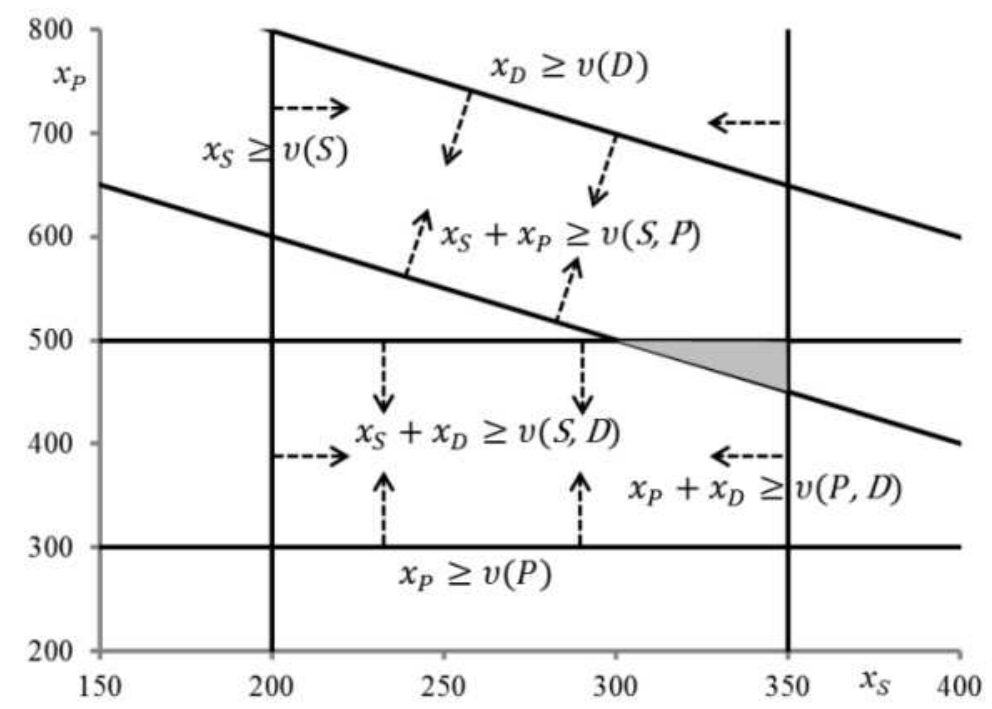

Figure 1. The core of the jazz band game (shaded area) is the set of imputations $\left(x_{S}, x_{P}, x_{D}\right)$, where $x_{D}=1000-x_{P}-x_{S}$, that obey the conditions of individual and collective rationality

Note that the core of the game is a set of all imputations that give every player and every coalition of players an incentive to participate in the grand coalition. In other words, to any payoff, that can be obtained by an individual player or a coalition of players there is the grand coalition 
that gives them the payoff which is greater or equal to that payoff. It is not necessarily the case in general. There are possible cases where players do not have an incentive to form the grand coalition - they are empty core games.

\section{Empty core game}

The core of the game can be empty. Let us assume that the characteristic function of some jazz band example is:

$$
\begin{array}{ccl}
\nu(\emptyset)=0 & \\
\nu(S)=200 & \nu(P)=300 & \nu(D)=0 \\
\nu(S, P)=800 & \nu(S, D)=500 & \nu(P, D)=650 \\
& \nu(S, P, D)=950 &
\end{array}
$$

i.e. the only difference is the grand coalition value $\nu(S, P, D)=950$. For such a modified game the core is empty. Indeed substituting the imputation condition $x_{P}+x_{S}+x_{D}=\nu(S, P, D)=950$ to collective rationality conditions yields that $x_{P} \leq 450, x_{S} \leq 300$ and $x_{D} \leq 150$, but such a triple $\left(x_{P}, x_{S}, x_{D}\right)$ is not a imputation since $x_{P}+x_{S}+x_{D}<\nu(S, P, D)$. Note that such a modified game is still superadditive. Nevertheless, the players do not find an incentive to form the grand coalition. One can see it, when assuming that players agree to form one of the possible duo coalitions. The collective rationality implies that their payoffs in duo coalitions are at least $x_{S}^{D}+x_{P}^{D}=800, x_{S}^{D}+x_{D}^{D}=500, x_{P}^{D}+x_{D}^{D}=650$. After solving the system of the three equations with unknowns $x_{P}^{D}, x_{S}^{D}, x_{D}^{D}$, one gets:

$$
\begin{aligned}
& x_{S}^{D}=325 \\
& x_{P}^{D}=475 \\
& x_{D}^{D}=175
\end{aligned}
$$

It means, that if a player chose the duo coalition, he could obtain at least one of the above payoffs (see also Fig. 2.) But the sum of them is $x_{S}^{D}+x_{P}^{D}+x_{D}^{D}=975>\nu(S, P, D)$ - greater than the amount accessible to the grand coalition. Therefore in duo solutions the players can have higher payoffs than the grand coalition but remember that one of the players must be excluded from the band! It is important not to be omitted.

It is interesting to simulate the possible negotiation scheme, which leads to a duo coalition and leaves one of them outside. Let us assume that we negotiate as the Pianist. If e.g. $P$ would like to get $x_{P}=480$ then his offer 


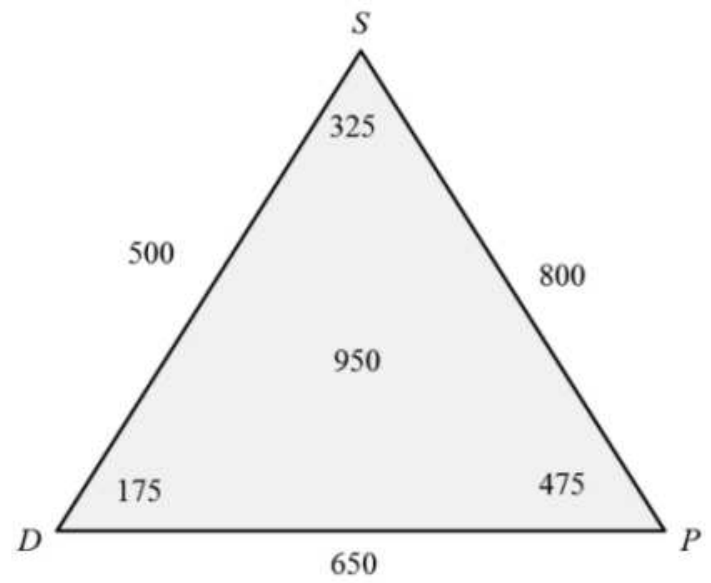

Figure 2. Offers that cannot be readily refused are the numbers in the corners of the triangle. They add up to values of each duo coalitions (at the sides of the triangle). In case of game with an empty core the sum of OCRR is greater than the grand coalition payoff 950.

to $S$ would be $x_{S}=320$, then $S$ can build coalition with $D$, sharing their duo payoff $x_{S}=325, x_{D}=175$, which is better than the proposal from $P$. Note that $D$ 's payoff is also better than $x_{D}=170$ that he could get if $P$ insisted on $x_{P}=480$. However, if $P$ 's offer to $S$ would be $x_{S}=325$, or to $D x_{D}=175$ (while $x_{P}=475$ ) neither $S$ nor $D$ can refuse it, because their duo payoff is exactly $\nu(S, D)=500=325+175$. The set of solutions (4) are called offers that cannot readily be refused [OCRR] (Raiffa, Richardson, $\&$ Metcalfe, Negotiation Analysis. The Science and Art of Collaborative Decision Making, 2007, p. 438).

The bargaining is in this case very unstable. By this we mean that there are no rational reasons to form one or another duo coalition. The threat that one of the players can be left outside the coalition may prevail the bargaining procedure resulting in acceptance of the grand coalition, which is less favourable but more secure for players. In the next section we attempt to find a fair share in case of the grand coalition.

\section{The Shapley value}

In this section we return to the original problem (1) of a nonempty core. We will try to compare different shares of the grand coalition payoff, which is in this case the most beneficial for the players. 
The first idea is the use of proportionality to OCRR. The concept of OCRR is independent of the size of the core and can be used to find a fair share. As we have noticed in the previous section, the sum of jazz band OCRR's (4) is 975 and it is smaller than the grand coalition payoff which is 1000 . The proportional rescaling of the shares (3) yields

$$
\begin{aligned}
& x_{S}^{P} \simeq 333.3 \\
& x_{P}^{P} \simeq 487.2 \\
& x_{D}^{P} \simeq 179.5
\end{aligned}
$$

The share proportional to OCRR has a disadvantage - it is not dependent on the individual payoffs of the players $\nu\left(p_{i}\right)$. Therefore it is easy to find conditions under which this solution is outside the core. It is enough to take $\nu(P)=490$ in (1) to obtain a nonempty core, which does not contain the share proportional to OCRR.

The Shapley value was first formulated in (Shapley, 1953). It is based on three axioms that are believed to reflect the symmetry of the problem well. Shapley proved that there is one and only one function $S V$, which to any game $(N, \nu)$ assigns an imputation $x=\left(x_{1}, x_{2}, \ldots, x_{n}\right)$ and obeys the axioms:

Axiom 1. The imputation $S V=\left(x_{1}, x_{2}, \ldots, x_{n}\right)$ depends only on the characteristic function $v$ and reflects its symmetries, e.g. if players $i$ and $j$ have symmetric roles then $x_{j}=x_{j}$.

Axiom 2. If $\nu(C)=\nu\left(C \backslash\left\{x_{i}\right\}\right)$ for all coalitions $C \subseteq N$, then $x_{i}=0$. The value of the null player is zero.

Axiom 3. $S V(\nu+\mu)=S V(\nu)+S V(\mu)$, where $S V(\nu+\mu)$ is the Shapley value of the sum game, defined for the same set of players $N$, whose payoffs are $(\nu+\mu)(C)=(\nu)(C)+(\mu)(C)$ for any coalition $C \subseteq N$.

The Shapley method is effective i.e. apart from the proof of existence it also gives an effective formula for calculating $S V$. The formula can be easily presented in the jazz band example, see Table 1.

In the rows of the table are defined different orders in which the grand coalition can be formed. The values added by the players in a given row of the Table 1 are calculated as follows (the row $P, D, S$ is explained):

$$
\begin{gathered}
P: \nu(P)-\nu(\emptyset)=300-0=300 \\
D: \nu(P, D)-\nu(P)=650-300=350 \\
S: \nu(S, P, D)-\nu(P, D)=1000-650=350
\end{gathered}
$$

In the last row of the table the average of each column is calculated, which is the Shapley value corresponding to each player. One can think about the Shapley value that it is the average value that each player adds 
Table 1

The calculation of the Shapley Value for the jazz band game (1)

\begin{tabular}{|c|c|c|c|c|}
\hline \multirow{2}{*}{ Ordering } & \multicolumn{3}{|c|}{ Value added by } & \multirow{2}{*}{ Total } \\
\cline { 2 - 4 } & $S$ & $P$ & $D$ & \\
\hline$S, P, D$ & 200 & 600 & 200 & 1000 \\
$S, D, P$ & 200 & 500 & 300 & 1000 \\
$P, S, D$ & 500 & 300 & 200 & 1000 \\
$P, D, S$ & 350 & 300 & 350 & 1000 \\
$D, S, P$ & 500 & 500 & 0 & 1000 \\
$D, P, S$ & 350 & 650 & 0 & 1000 \\
\hline Average: & 350 & 475 & 175 & 1000 \\
\hline
\end{tabular}

while the grand coalition is formed, the average is taken over all the possible orders in which the players can enter that coalition. Note that Shapley value reflects the payoffs (1) of single, duo and trio bands. The values of the share proportional to OCRR and Shapley value are shown in Fig. 3.

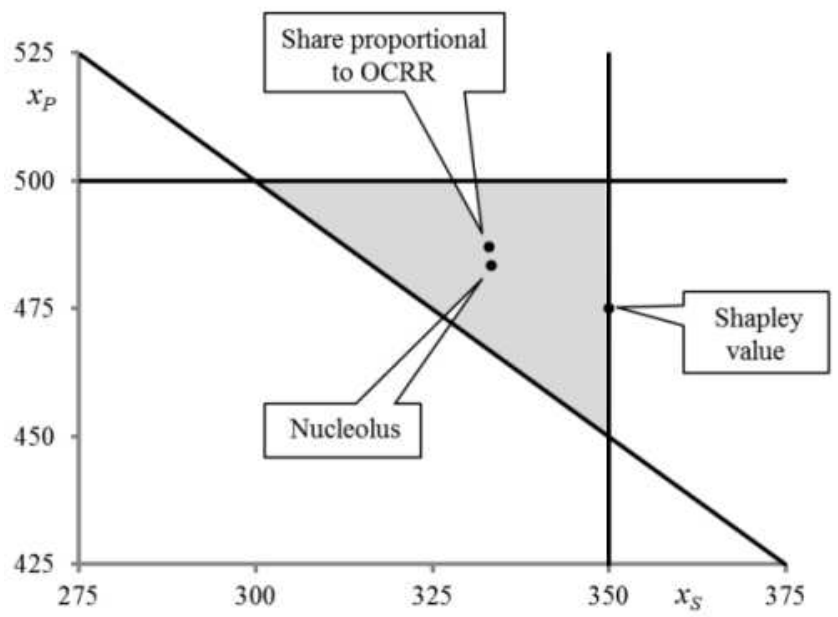

Figure 3. The core of the jazz band game (1) with different shares for the grand coalition

It may be surprising that the share proportional to OCRR lies closer to the center of the core than the Shapley value, which in this case lies at its border. Indeed the share proportional to OCRR is closer to the center of the core because, in this case, it is defined by the collective rationality conditions. The Shapley value therefore may not be satisfactory for the coalition $(P, D)$ whose total Shapley value $475+175=650$ is the same as in case of their duo coalition $\nu(P, D)=650$. 
The Shapley value has also other drawbacks as well - it may yield inconsistent answers. If e.g. two players have payoffs of 200 and 300 and their coalition value is 300 then the Shapley value is 100 for the first player and 200 for the second ond one. If we duplicate the problem i.e. there are four players, two receive payoffs of 200 and two - payoffs of 300 and the grand coalition payoff is 600 , then we would expect the Shapley value to be duplicated: it should be 100 for the first two players and 200 for the other two players. But Shapley value allocates $116 \frac{2}{3}$ - to each of the first two players and $182 \frac{1}{3}$ - for each of the remaining two players (Young, 1994). Such a solution is inconsistent with the case of two players. Note that the Axiom 3 does not prevent such a drawback because it concerns only games with the same number of players.

\section{The nucleolus}

In the previous section we have defined two "solutions" to the jazz band game, the imputation proportional to OCRR and the Shapley value. Both of them have drawbacks and may not be regarded as a fair share in case of that game. In the present section we are going to define one more value, nucleolus - introduced by (Schmeidler, 1969).

Let us recall that the core of the game consists of imputations that satisfy $\sum_{p_{i} \in C} x_{i} \geq \nu(C)$ for any coalition $C \subseteq N$. In case of the jazz band game the core is not empty, and our goal is to find one imputation $\boldsymbol{x}=$ $\left(x_{1}, x_{2}, \ldots, x_{n}\right)$ that is fair and in particular - is located as close as possible to its center (because of the collective rationality condition). The way to obtain such a value is to maximize the distance of the imputation from the sides of the core. The measure of its distance is the value

$$
d_{C}(\boldsymbol{x})=\sum_{p_{i} \in C} x_{i}-\nu(C)
$$

for $C \subseteq N$ corresponding to different coalitions defining lines forming the core. Some of these lines form sides of the core and some of them lie outside the core. Our goal is to find the imputation $\boldsymbol{x}$, which will maximize the minimal of the distances $d_{C}(\boldsymbol{x})$. In other words, we are looking for the imputation that is as far as possible from the sides of the core - therefore maximizing the minimal amount that players earn in addition to the amount they can have without forming the great coalition. In some cases there are several different imputations that maximize the minimal distance. Then 
one should maximize the second minimal, then the third minimal, and so on until one gets the unique imputation.

Let us start from the Shapley value and calculate the distances

$$
\begin{gathered}
d_{S}(\boldsymbol{x})=x_{S}-\nu(S)=350-200=150 \\
d_{P}(\boldsymbol{x})=x_{P}-\nu(P)=475-300=175 \\
d_{D}(\boldsymbol{x})=x_{D}-\nu(D)=175-0=175 \\
d_{S, P}(\boldsymbol{x})=x_{S}+x_{P}-\nu(S, P)=350+475-800=25 \\
d_{S, D}(\boldsymbol{x})=x_{S}+x_{D}-\nu(S, D)=350+175-500=25 \\
d_{P, D}(\boldsymbol{x})=x_{P}+x_{D}-\nu(P, D)=475+175-650=0
\end{gathered}
$$

The distance corresponding to the grand coalition is zero by definition $\sum_{i=1}^{n} x_{i}=\nu(N)$. As one can notice, the minimal of the distances is $d_{P, D}(\boldsymbol{x})=0$ (because the Shapley value lies at the side of the core). To maximize the minimal distance we may increase values of $x_{P}$ and $x_{D}$ but we have to remember that $x_{S}=1000-x_{P}-x_{D}$. Therefore we have to make sure to do it only to such an extent, where $d_{S, P}(\boldsymbol{x})$ and $d_{S, D}(\boldsymbol{x})$ are not decreased too much. The solution is easy to find assuming equal minimal distances

$$
d_{S, P}(\boldsymbol{x})=d_{S, D}(\boldsymbol{x})=d_{P, D}(\boldsymbol{x})=16 \frac{2}{3}
$$

And the corresponding imputation - the nucleolus of the problem is

$$
\begin{aligned}
& x_{S}^{N}=333 \frac{1}{3} \\
& x_{P}^{N}=483 \frac{1}{3} \\
& x_{D}^{N}=183 \frac{1}{3}
\end{aligned}
$$

In Figure 3 we can see that nucleolus is the central point of the core - it is so by definition - the nucleolus have the same distances from three sides of the triangle forming the core. Therefore it can be regarded as the share that is most equitable among others. It has also the amiable feature that it is always in the core, provided it is not empty.

The nucleolus has another interesting characteristic feature - it can be defined even in case of an empty core. In this case some of distance formulas (8) give negative numbers. But the procedure of finding nucleolus is the same. It is the point $\boldsymbol{x}$ that maximizes the minimal of the distances $d_{C}(\boldsymbol{x})$ for all $C \subset N$. In case of the empty core game (3) the nucleolus is basically the same as in eq. (10), where each value is reduced by the same amount $16 \frac{2}{3}=\frac{50}{3}$, which comes from the condition $\sum_{i=1}^{n} x_{i}=\nu(N)=950$. 


\section{Jazz band negotiations}

In this section we will apply the obtained results to actual negotiations between the three artists. We are especially interested in preparation and in bargaining phase of negotiations. Firstly, let us notice, that the negotiation process will be qualitatively different depending on the core of the game. If the core is empty, the players may not find an incentive to form the grand coalition and one can expect that they agree to form one of the possible duo coalitions, leaving the third partner outside. The crucial point in negotiation would be to prevent being left outside the coalition. For a nonempty core, there is no point in leaving one of the players outside. The third coalition partner always brings enough value - the grand coalition payoff can be divided by imputations that obey any two-way collective rationality conditions.

Let us first assume the case of an empty core $\nu(S, P, D)=950$. As we remember, in such case the players have OCRR values (4), which are fair shares in any duo coalition. Each player wants to approach one of the others to encourage him to form a coalition. Because the share of $D$ is the smallest, he is most susceptible to incentives.

Let $S$ approach $D$ confidentially and offer him $x_{D}=180$ - five FF more than he can expect from any duo OCRR coalition. Assume that $S$ and $D$ agree to form a coalition and act together against $P$. Then they can approach $P$ to offer him a trio coalition but on their conditions. The value that $P$ is adding to $S$ and $D$ duo is 450 and his individual rationality level is $x_{P}=300$. So $S$ and $D$ coalition can offer $P$ half of the added value i.e. $x_{P}=375$, while keeping $x_{S}+x_{D}=575$, which can be divided e.g. $x_{S}=360$ and $x_{P}=215$. But because the core is empty any such trio coalitions can be outbid by a duo. For example $P$ can invite $D$ to a duo coalition $x_{P}=400$ and $x_{D}=250$ which would leave $S$ outside. Then $S$ can be invited to a three-way coalition by $P$ and $D$ under their conditions, and so on... On each level of such bargaining one or two of the players are left with the share which is less than their OCRR and one (or two) of them usually get more than OCRR. The player that is most likely to get a share exceeding his OCRR is the weakest player to whom such an offer is the most tempting. Therefore he can choose which competitor he wishes to form a coalition with. But his claims must be moderate as the other players can always build a coalition leaving him without any payoff. On the other hand the strongest player is most likely to be the one, who gets less than his OCRR value. Being potentially the strongest partner, he is most likely to be punished by his weaker partners. Therefore in the empty core case 
there is a natural tendency that the negotiation results change from OCRR towards egalitarian solutions.

The coalition game with an empty core was played in a series of experiments performed by the author with trainees attending negotiation courses. In these experiments three parties, not familiar with game theory, were given the scenario of the singer, the player and the drummer. The time was limited to 20 minutes for preparation and to 40 minutes for negotiations. The parties were asked to prepare for negotiations and propose their individual "fair share". After that, they started face to face negotiations in triples and pairs - they were encouraged to meet occasionally in pairs without the presence of the third party. The results confirm the above considerations. More than $80 \%$ of negotiations resulted in a grand coalition agreement. The average results of the three-way coalitions are shown in Table 2 in comparison with the Shapley value and the nucleolus. Note that OCRRs, which are also shown in the table, sum up to 975.

In about $90 \%$ of negotiations that ended in three-way coalitions, the players got involved in some duo coalitions at some time during the negotiations. In the $20 \%$ that did not result with a grand coalition, about half of them did not form any coalition at all and the other half formed some duo coalitions.

\section{Table 2}

The comparison of OCRR and experimental results with the Shapley value and nucleolus for the jazz band game with an empty core

\begin{tabular}{|l|c|c|c|c|}
\hline & $\mathrm{S}$ & $\mathrm{P}$ & $\mathrm{D}$ & $x_{P}+x_{S}+x_{D}$ \\
\hline OCRR & 325 & 475 & 175 & 975 \\
Experiment & 310 & 425 & 215 & 950 \\
Shapley value & 333,3 & 458,3 & 158,3 & 950 \\
Nucleolus & 316,7 & 466,7 & 166,7 & 950 \\
\hline
\end{tabular}

Two artists who form a two-way coalition (e.g. the singer and the pianist) could ask the nightclub owner to increase the payoff of the grand coalition from $950 \mathrm{FF}$ to $1000 \mathrm{FF}$. This relatively small increase changes the solutions of the game in a way that there is an incentive for everybody to form a grand coalition which is preferable also for the owner of the club. In case of a nonempty core there is no threat of being left outside the grand coalition and the players are much more likely to agree to Shapley value or nucleolus. Therefore while the singer and pianist may wish for the trio payoff to be increased, the drummer may not be interested in such a change. 


\section{Conclusions}

To summarize, we have proposed three different ways to calculate the solution of the jazz band game assuming that players create a grand coalition: share proportional to OCRR, Shapley value and nucleolus. In the game with a nonempty core the first and the third are located close to the center of the core. The Shapley value lies exactly at the side of the core and is far from its center.

In case of the game with an empty core the most profitable solutions are obtained by two-way coalitions. The threat to one of the players to be left out of a two-way coalition introduces coalitional instabilities - which duo coalition is to be formed? The most likely partner in two-way coalitions is the weakest partner. As a result of that the weakest partner gains more than his OCRR value and the coalition is extended to a three-way but this happens at the expense of one of the other partners. We have confirmed this result in a series of negotiations conducted by experiment participants. The weakest player may not have an incentive to increase the three-way coalition payoff to the level of a nonempty core.

In case of a nonempty core the Shapley value is located at the side of the core - far from its center. Note, however, that only the Shapley value takes into account both individual and duo coalition payoffs. In case of the share proportional to OCRR the individual payoffs are neglected. The same is true in the case of nucleolus - conditions connected with individual payoffs (three first equations in (8)) do not influence its position. That is the reason why, in our opinion, the Shapley value, although it may not be in the center of the core, is still a very good answer to ensure a fair solution of the three players problem.

It could be interesting to modify the nucleolus by redefining the minimal distances (collective rationality) in a way dependent on the individual rationality conditions.

\section{Acknowledgments}

This work was partially supported by the grant from Polish National Science Center DEC-2011/03/B/HS4/03857.

\section{R E F E R E N C E S}

Malawski, M., Wieczorek, A., \& Sosnowska, H. (1997). Konkurencja i kooperacja. Teoria gier $w$ ekonomii i naukach spolecznych. Warszawa: Wydawnictwo Naukowe PWN. 
Raiffa, H., Richardson, J. i Metcalfe, D. (2002). Negotiation Analysis. The Science and Art of Collaborative Decision Making. Cambridge, London: The Belknap Press of Harvard University Press.

Raiffa, H., Richardson, J., \& Metcalfe, D. (2007). Negotiation Analysis. The Science and Art of Collaborative Decision Making. Cambridge, London: The Belknap Press of Harvard University Press.

Roth, A. (1988). The Shapley value. Essays in honor of Lloyd S. Shapley. In A. Roth (Ed.). Cambridge: Cambridge University Press.

Schmeidler, D. (1969). The nucleolus of the characteristic function game. SIAM Journal on Applied Mathematics, 17, pp. 1163-1170.

Shapley, L. (1953). A value for n-person games. W K. Tucker (Red.), Contributions to the theory of games II (pp. 307-317). Princeton: Princeton University press.

Von Neumann, J., \& Morgenstern, O. (1944). Theory of Games and Economic Behavior. Wiley.

Young, P. (1994). Equity in Theory and Practice. Princeton: Princeton University Press. 